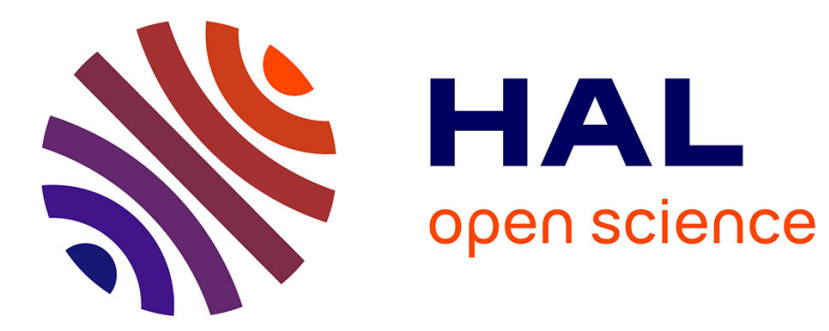

\title{
Calibration for polarization filter array cameras: recent advances
}

Yilbert Giménez, Pierre-Jean Lapray, Alban Foulonneau, Laurent Bigué

\section{To cite this version:}

Yilbert Giménez, Pierre-Jean Lapray, Alban Foulonneau, Laurent Bigué. Calibration for polarization filter array cameras: recent advances. Fifteenth International Conference on Quality Control by Artificial Vision, May 2019, Mulhouse, France. pp.79, 10.1117/12.2521752 . hal-02187718

\section{HAL Id: hal-02187718 \\ https://hal.science/hal-02187718}

Submitted on 18 Jul 2019

HAL is a multi-disciplinary open access archive for the deposit and dissemination of scientific research documents, whether they are published or not. The documents may come from teaching and research institutions in France or abroad, or from public or private research centers.
L'archive ouverte pluridisciplinaire HAL, est destinée au dépôt et à la diffusion de documents scientifiques de niveau recherche, publiés ou non, émanant des établissements d'enseignement et de recherche français ou étrangers, des laboratoires publics ou privés. 


\title{
Calibration for polarization filter array cameras: Recent Advances
}

\author{
Yilbert Giménez $^{\mathrm{a}}$, Pierre-Jean Lapray ${ }^{\mathrm{a}}$, Alban Foulonneau ${ }^{\mathrm{a}}$, and Laurent Biguéa \\ aIRIMAS, EA7499, Université de Haute-Alsace, Mulhouse, France
}

\begin{abstract}
A Polarization Filter Array (PFA) camera is an imaging device capable of analyzing the polarization state of light in a snapshot way. These cameras exhibit spatial variations, i.e. nonuniformity, in their response due to optical imperfections introduced during the nanofabrication process. Calibration is done by computational imaging algorithms to correct the data for radiometric and polarimetric errors. In this paper, we review existing calibration procedures, and show a practical implementation result of one of these methods applied to a commercially available PFA camera.
\end{abstract}

Keywords: Polarization Filter Array, division-of-focal-plane polarimeter, calibration, imaging, noise correction

\section{INTRODUCTION}

The electric field that describes an electromagnetic radiation can be described by a vector, whose direction of oscillation is perpendicular to the direction of the wave. This geometrical description of waves is known as polarization. ${ }^{1}$ Reptiles or birds, for examples, are capable to perceive polarized information, whereas humans, like any other mammals, are only sensible to two properties of light, intensity and color. The analysis of the polarization scattered by a scene is known as polarimetric imaging; it yields a complementary performance to classical intensity imaging. ${ }^{2}$ A mathematical tool named Stokes vector is used to describe practically and efficiently the modification of the polarization states when the light travels and interacts with different materials. Polarization analysis using polarimeter instruments becomes increasingly popular in imaging applications such as classification of materials, ${ }^{3}$ 3D inspection and reconstruction, ${ }^{4}$ image dehazing, ${ }^{5}$ etc.

In general, polarimeters can be categorized into scanning or snapshot devices, none of which is exempt of tradeoffs and optical imperfections. Scanning polarimeters include the Division-of-Time polarimeter (DoT), whereas snapshot polarimeters include the Division-of-Amplitude (DoAmP), Division-of-Aperture (DoA) and Divisionof-Focal-Plane (DoFP). ${ }^{6,7}$ Despite the fact that each approach is arguably suitable for different applications, DoFP is the ideal choice for real-time imaging as it is capable to analyze light polarization within one sensor integration period, which avoids motion artifacts introduced by DoT polarimeters. These devices lead to a lower cost, simpler design, higher compactness than other snapshot devices.

The DoFP polarimeters rely on the cell-to-cell coupling of a Polarizer Filter Array (PFA) to the imaging system's focal-plane array. PFA technology is a derivative of the filter array imaging principle, ${ }^{8,9}$ and was first patented in $1995,{ }^{10}$ but most of the practical implementations and technology advances have been made since 2009. Manufacturing processes are various, ${ }^{11}$ but commercial sensors (like the SONY IMX250 MZR sensor) tend to have a standardized spatial arrangement with a repeating pattern of four linear polarizers with orientation axes of $0,45,90$ and 135 degrees $^{12}$ (see Figure 1). Every quadrant is located diagonally to its orthogonal counterpart, even if it is not obvious that this spatial arrangement is optimal. ${ }^{13}$ The acquisition of intensities through the four linear polarizers makes it possible the estimation of the first three Stokes vector components of the input light. To sense circular polarization, i.e. the fourth Stokes component, additional optical elements must be combined with the PFA. Practical implementation of full-Stokes PFA instruments is at its very beginning. In this paper, we will only consider the linear analysis of polarization.

Further author information: (Send correspondence to Y.G.)

Y.G.: E-mail: yilbert.gimenez-henriquez@uha.fr

P.-J.L.: E-mail: pierre-jean.lapray@uha.fr 
Intrinsically, a silicon sensor (CMOS or CCD) has several sources of errors. Dark current, readout or saltand-pepper noises are typical examples. They are corrected using a particular noise pattern where pixels are susceptible to give brighter intensities than others when illuminated with a homogeneous light. Filter array imaging also suffers from sparsity, i.e. each pixel senses only one polarization channel, which introduces instantaneous field-of-view errors ${ }^{11}$ when reconstructing $2 \mathrm{D}$ polarization scene information from sparse data. PFA cameras exhibit the noises inherent to silicon sensors, but additional noises due to manufacturing optical imperfections of filters are introduced. Thus, each polarizer has its own optical characteristics, i.e. transmission, diattenuation and polarization analysis orientation. ${ }^{14}$ It results in a pattern noise that could lead to a spatial variation of digital values up to $20 \%$ over the whole sensing area. ${ }^{15}$ Spatial calibration procedure is thus necessary to compensate for these nonuniformities.

In this paper, we will introduce the global PFA imaging model in Section 2, before reviewing calibration procedures in Section 3. Results of an implementation of one of the calibration procedure will be shown, along with discussion and conclusion in Section 4.

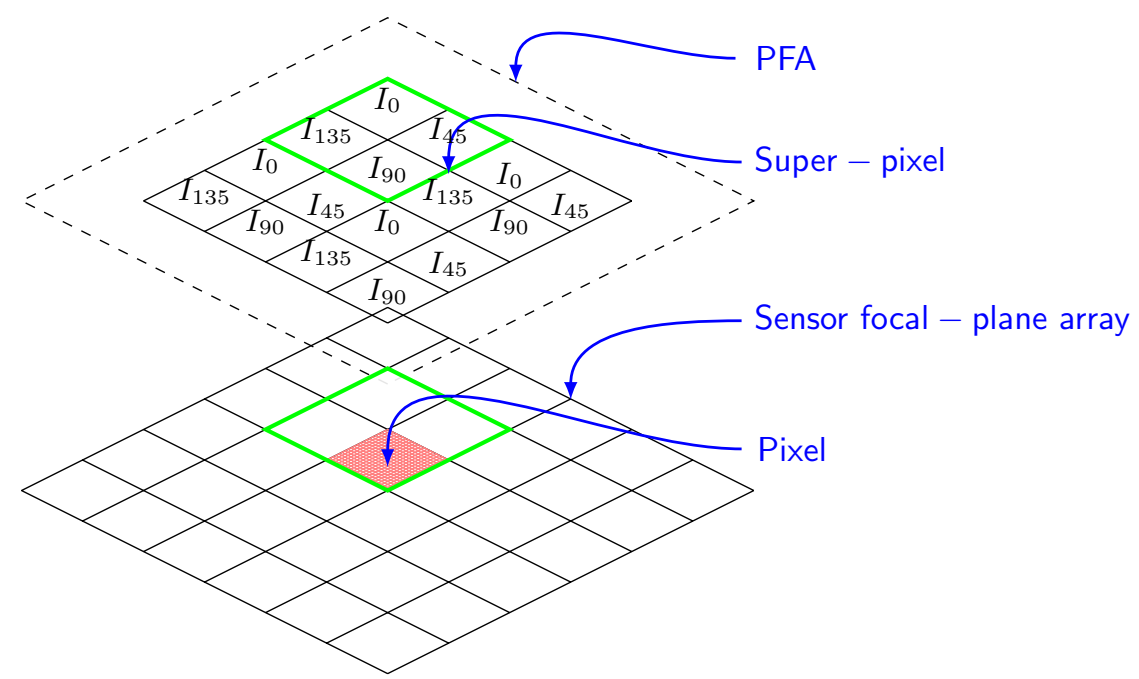

Figure 1. The camera design considered in this paper is a polarization filter assembly over a monochrome sensor. The sensor matrix is composed of photodiodes and each polarization filter covers one sensor pixel.

\section{PFA IMAGING MODEL}

In this section, we enunciate the polarization measurement model coming with PFA cameras. Stokes formalism includes a suitable depiction of polarization states of the light. Four components, arranged in a vector, fully describe the polarization states of light. Since a PFA polarimeter is intrinsically a linear polarization analyzer composed by linear diattenuators, the input Stokes vector of light $\mathbf{S}_{\text {in }}$ that reaches the camera can be simplified as $\mathbf{S}_{\mathbf{i n}}=\left[\begin{array}{llll}S_{0} & S_{1} & S_{2} & 0\end{array}\right]^{t}$. Mueller matrix $\mathbf{M}$ which is a $4 \times 4$ matrix, describes the alteration of polarization characteristics of a material element, it can be seen as a transfer function of Stokes vectors. A Stokes vector is linearly transformed such as $\mathbf{S}_{\text {out }}=\mathbf{M S}_{\mathbf{i n}}$.

A photodetector array transduces luminous intensity into a camera response $I_{i}$, where $i$ is the spatial index over pixels. Thus, only the top row of the Mueller matrix $\mathbf{M}$ is useful to know, which is also called the pixel's analysis vector $\mathbf{A}_{\mathbf{i}}=\left[\begin{array}{llll}a_{0, i} & a_{1, i} & a_{2, i} & 0\end{array}\right]$. Thus, if all the first rows of the corresponding Mueller matrices in the sensing area are known, the errors due to nonideal characteristics can be mitigated through calibration. In other words, to carry out precise measurements, the filters do not need to be ideal.

We could now define the imaging model that transforms the incoming Stokes vector into a per-pixel sensed value as follows:

$$
I_{i}=\mathbf{A}_{\mathbf{i}} \mathbf{S}_{\mathbf{i n}}
$$


A PFA camera has four different polarization angles of analysis, arranged in a $2 \times 2$ pattern. We could then assume a unique spatial position for the four adjacent polarizers, and group the four analysis vectors into an array of vectors (the measurement matrix) that we call $\mathbf{W}_{\mathbf{j}}$, where $j$ indexes the super-pixel spatial position. Equation 1 could be extended to this specific configuration:

$$
\mathbf{I}_{\mathbf{j}}=\left[\begin{array}{c}
I_{j, 0} \\
I_{j, 45} \\
I_{j, 90} \\
I_{j, 135}
\end{array}\right]=\mathbf{W}_{\mathbf{j}} \mathbf{S}_{\mathbf{i n}}=\left[\begin{array}{c}
\mathbf{A}_{\mathbf{j}, \mathbf{0}^{\circ}} \\
\mathbf{A}_{\mathbf{j}, \mathbf{4 5}} \\
\mathbf{A}_{\mathbf{j}, \mathbf{9 0}} \\
\mathbf{A}_{\mathbf{j}, \mathbf{1 3 5}}
\end{array}\right] \mathbf{S}_{\mathbf{i n}}
$$

Considering Eq. 2, the input Stokes vector can be estimated from intensities using the pseudo-inverse of $\mathbf{W}_{\mathbf{j}}$, assuming that $\mathbf{W}_{\mathbf{j}}$ is known. In most cases, $\mathbf{W}_{\mathbf{j}}$ matrices are not given by PFA manufacturers, and should be estimated during a calibration step. We will see in the next section how this model is investigated or evenly extended in the literature to perform calibration of PFA camera.

\section{CALIBRATION TECHNIQUES}

\subsection{Assumptions \& General Procedure}

Most of the techniques presented in this paper have several important assumptions about the sensor, the calibration optical setup, and the statistical behaviour of signal. One assumes that:

- The sensor operates in the linear regime. Most of the calibration techniques also assume that there is no cross-talk effect when coupling the PFA with the focal-plane array, and moreover may vary along with the f-number of the lens.

- There is no spectral dependency on $\mathbf{W}$, i.e. retardance is flat over the range of wavelengths considered, and the reference polarizer used to generate training Stokes vectors is perfect (no diattenuation, retardance or transmission problems),

- The acquisition during calibration is not corrupted by temporal noise (a mix of Poisson and Gaussian noises), and that there is no need to apply flat-field procedure to correct training data for residual illuminant spatial deviation.

All the calibration techniques for PFA that we will present share the same global procedure :

1. Inputting a set of light stimuli (training data) with known Stokes vectors to the polarimeter,

2. For each input Stokes stimuli, capture a series of images and average/add them,

3. Estimate the $\mathbf{A}$ vectors or $\mathbf{W}$ matrices, i.e. the polarization properties at each pixel or super-pixel, by solving an inverse problem,

4. Compute the gains and offsets from Step 3., and apply them to correct raw values.

\subsection{Single-Pixel Calibration}

Single-pixel calibration is the way to calibrate each pixel independently, without considering the polarization properties of its neighborhood. Powell and Gruev ${ }^{15}$ added the offset noise $d_{i}$ in the model in Equation 1, to take into account for additive noise during calibration:

$$
I_{i}=\mathbf{A}_{\mathbf{i}} \mathbf{S}_{\mathbf{i n}}+d_{i}
$$

Then, a calibration function is applied to pixels as :

$$
I^{\prime}{ }_{i}=g_{i}\left(I_{i}-d_{i}\right)
$$

where $I_{i}^{\prime}$ is the corrected value and $g_{i}=\frac{\left\|\mathbf{A}_{\text {ideal }}\right\|}{\left\|\mathbf{A}_{\mathbf{i}}\right\|}$ is the normalized gain, where $\mathbf{A}_{\text {ideal }}$ and $\mathbf{A}_{\mathbf{i}}$ vectors are assumed to be co-linear. As stated in Powell and Gruev, ${ }^{15}$ this assumption implies that only transmission errors can be compensated, the single-pixel calibration doesn't correct for diattenuation and orientation variations (rotational offset) across the PFA structure. Thus, the single-pixel method yields errors when calculating the angle and degree of polarization. 


\subsection{Super-Pixel Calibration}

Super-pixel calibration is a more evolved technique. It uses a relative neighborhood of four pixels to form the super-pixel used in the calibration framework; it compensates for deviations in transmission, diattenuation and orientation. AOLP (Angle Of Linear Polarization) and DOLP (Degree Of Linear Polarization) are much more precise with this technique since a in-common pixel correction is done instead of individual.

Myhre et al. ${ }^{16}$ use Eq. 2 to calibrate their full-Stokes polarimeter. They assume no additive noise in their model. On the contrary, Powell and $\mathrm{Gruev}^{15}$ include additive noise in their super-pixel calibration procedure:

$$
\mathbf{I}_{\mathbf{j}}=\mathbf{W}_{\mathbf{j}} \mathbf{S}_{\mathrm{in}}+\left[\begin{array}{c}
\mathrm{d}_{\mathrm{j}, \mathbf{0}^{\circ}} \\
\mathrm{d}_{\mathbf{j}, \mathbf{4 5 ^ { \circ }}} \\
\mathbf{d}_{\mathbf{j}, \mathbf{9 0 ^ { \circ }}} \\
\mathrm{d}_{\mathrm{j}, \mathbf{1 3 5 ^ { \circ }}}
\end{array}\right]
$$

As for the single-pixel, a calibration function is applied as follows:

$$
\mathbf{I}_{\mathbf{j}}^{\prime}=\mathbf{g}_{\mathbf{j}}\left(\mathbf{I}_{\mathbf{j}}-\mathbf{d}_{\mathbf{j}}\right)
$$

where $\mathbf{g}_{\mathbf{j}}=\mathbf{W}_{\text {ideal }} \mathbf{W}_{\mathbf{j}}^{+}$gathers the four normalized gains recovered by pseudo-inverse operation over $\mathbf{W}_{\mathbf{j}}$.

They found that the super-pixel calibration method reduces reconstruction error, in terms of RMSE for DOLP and AOLP, by a factor of around ten compared to the single-pixel calibration method along with correction of diattenuation and orientation.

\subsection{Adjacent Super-Pixel Calibration}

Chen et al. ${ }^{17}$ performed a complementary strategy to the super-pixel calibration, by adding a computational step at the end the super-pixel algorithm. Once Eq. 6 is applied, every single-pixel is recalibrated as a function of the weighted average of the four overlapped super-pixels neighborhood at the pixel position:

$$
I_{j}^{\prime \prime}=1 / 4\left[I_{j}^{\prime}+I_{j l e f t}^{\prime}+I_{j \text { diag }}^{\prime}+I_{j a b o v e}^{\prime}\right]
$$

where the terms of average are the calibrated values of the super-pixels located to the left, above and diagonal (adjacent to the latter two) respectively. Authors do not give explanation or justification about doing this average. They argue that the error between the acquired DoLP and the ideal value is reduced in range by a factor of ten. In the same way, the error for the AoLP is reduced in range by a factor of four. They also show visually more uniformity in their reconstructed DOLP and AOLP parameters. When applied to a real image, they show that the calibration improves polarization feature contrasts in both intensity and DoLP images. No comparison with the state-of-art is done.

\subsection{Average Analysis Matrix Calibration}

Zhang et al. ${ }^{18}$ implement the same sequence as Chen et al. ${ }^{17}$ with the same neighborhood, i.e. by calculating the analysis matrix and the offsets from training data. But, instead of using an ideal analysis matrix to produce the multiplicative adjustment of the calibration function, an all-encompassing PFA average matrix is used as the common factor in Eq. 6 such that $\mathbf{g}_{\mathbf{j}}=\mathbf{W}_{\text {mean }} \mathbf{W}_{\mathbf{j}}^{+}$. No analytic criteria are stated for using the mean matrix but this value is closed to the ideal. They apply the calibration to a real image and show qualitative results; the edges are smoother compared to the super-pixel method. Quantitatively, they found a thousandth order of magnitude reduction of the RMS error between the mean DoLP and the pixels DoLP. A quantitative comparison with the super-pixel calibration ${ }^{15}$ is done using real images, but the authors do not specify how the mean DoLP of an object under study is sampled out of the image.

\subsection{Installation Calibration}

Han et al. ${ }^{19}$ proposed another calibration method in agreement with the super-pixel process by Powell and Gruev $^{15}$ along with an error model associated with cross-talk from neighboring pixels. The main difference lies in the error model of the sensor as the model takes into account any potential misalignment between the PFA and the focal-plane array due to installation error and limitation of mechanical accuracy. Installation errors include translation deviation and angular misalignment. 


\subsection{Variance Estimation and Rapid Calibration}

Hagen $e t a l .{ }^{20}$ put forward a single-pixel calibration approach with simpler calibration setup than prior calibration methods. ${ }^{15,17-19}$ They use only four measurements to characterize each pixel individually, and recover the incident intensity, the orientation axis of analysis, and the diattenuation parameters. On the one hand, an angle-graduated diffuser replaces the integrating sphere and the rotational stage. As their calibration formulas does not depend on the incident light intensity, there is no need to have spatially uniform lightning at the input. On the other hand, they define a measurement statistical model to estimate parameter variances and calibration accuracy. It allows to separate efficiently the temporal noise from the spatial noise. By doing this, it avoids the need of averaging a lot of samples to partially cancel the temporal noises when using conventional calibration. Different camera calibrations could lead to different measurement noises, the method allows to compare qualitatively different cameras. Nevertheless, no evaluation is done relatively to other calibration methods. Another contribution from this paper is a great discussion about the extinction ratio: the more the extinction ratio, the higher calibration accuracy is needed.

\section{DISCUSSION AND CONCLUSION}

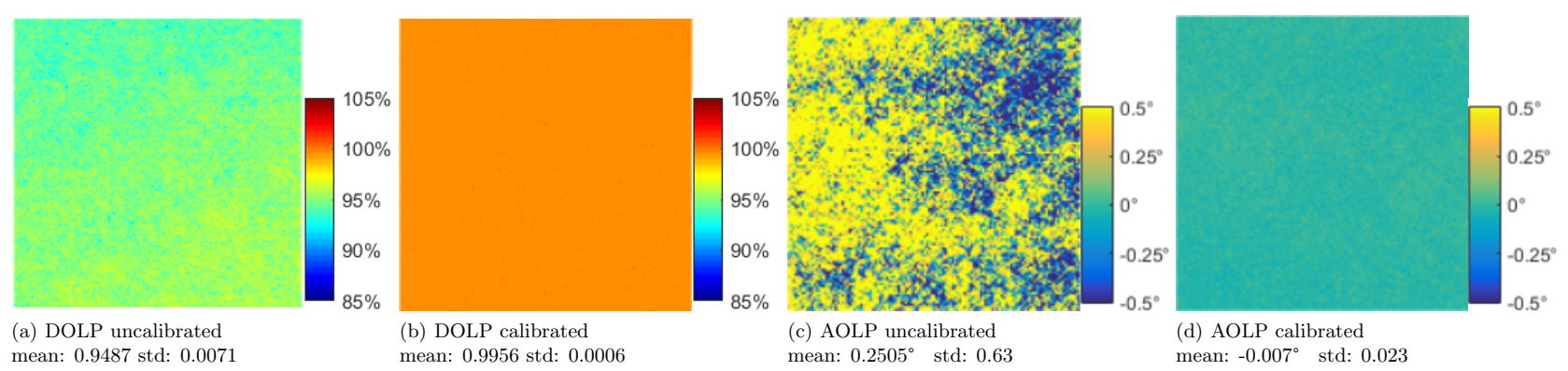

Figure 2. Zero-oriented fully-polarized light capture and parameter estimations. The region of interest is an area of $300 \times 300$ pixels in the center of the sensor.

An implementation of the super-pixel calibration from Powell and Gruev ${ }^{15}$ is demonstrated here. We used the same acquisition procedure and setup, but with different instruments. The adjustable shutter is substituted by the camera's integration time. Illuminant light source is provided by an Intralux 4000 module. The light passes through a Thorlabs IS200 integrating sphere to generate nominally uniformed unpolarized light. A LPVISE100A linear polarizer from Thorlabs is rotated using a rotational stage to generate the input Stokes vector. Lastly, light enters the PFA camera from 4D Technology with a Sony IMX174 CMOS sensor.

Figure 2 shows pseudo-color images representing the DOLP and AOLP before and after super-pixel calibration at zero degree. It is seen that, when the image is uncalibrated, the DOLP has a mean value significantly lower than 1. The color scale allows to intuitively visualize the heterogeneity of the values in the flat-field. The mean AOLP is improved by a shifting near the ideal value and its standard deviation is improved by a factor of 25 . We believe that this improvement is necessary for numerous of applications. The uncalibrated camera values could lead to false contrasts or large errors when computer vision algorithms are applied, e.g. material inspection, shape from polarization, index of refraction retrieval, or illuminant direction estimation.

To conclude, it is not obvious that calibration will enhance the polarimetric data in all scene conditions. It is clearly stated that the predominant source of noise in a PFA camera is the spatial noise in several cases. But when temporal noise is too high or when dealing with high polarizing scenes, e.g. for active imaging, the improvement brought through a more evolved calibration than the super-pixel method could be insignificant. This is discussed in a recent paper by Roussel et al. ${ }^{21}$ It is also stated in all of the reviewed papers that we should recalibrate the polarimeter when changing the f-number of the lens due to cross-talk. Recent sensors put the filter array below the micro lenses, which mitigates this effect.

Future works would be to find experimental and evaluation methods in order to benchmark the different calibration models extensively. Moreover, a complete PFA camera pipeline for high dynamic range enhancement 
based on multiple exposure times ${ }^{22}$ (to correct for saturation and uniformity of noise among channels), is missing in the literature.

\section{REFERENCES}

[1] Feynman, R. P., Leighton, R. B., and Sands, M., "The feynman lectures on physics; vol. i," American Journal of Physics 33(9), 750-752 (1965).

[2] Goudail, F. and Tyo, J. S., "When is polarimetric imaging preferable to intensity imaging for target detection?," J. Opt. Soc. Am. A 28(1), 46-53.

[3] Tominaga, S. and Kimachi, A., "Polarization imaging for material classification," Optical Engineering 47(12), 1 - $14-14$ (2008).

[4] Ferraton, M., Stolz, C., and Mériaudeau, F., "Optimization of a polarization imaging system for 3d measurements of transparent objects.," Opt. Express 17(23), 21077-21082.

[5] Schechner, Y. Y., Narasimhan, S. G., and Nayar, S. K., "Polarization-based vision through haze," Appl. Opt. 42(3), 511-525.

[6] Lapray, P.-J., Gendre, L., Foulonneau, A., and Bigué, L., "An fpga-based pipeline for micropolarizer array imaging," International Journal of Circuit Theory and Applications 46(9), 1675-1689 (2018).

[7] Tyo, J. S., Goldstein, D. L., Chenault, D. B., and Shaw, J. A., "Review of passive imaging polarimetry for remote sensing applications," Appl. Opt. 45(22), 5453-5469.

[8] Lapray, P.-J., Wang, X., Thomas, J.-B., and Gouton, P., "Multispectral filter arrays: Recent advances and practical implementation," Sensors 14(11), 21626 (2014).

[9] Lapray, P.-J., Thomas, J.-B., and Gouton, P., "A multispectral acquisition system using msfas," Color and Imaging Conference 2014(2014), 97-102 (2014-11-03T00:00:00).

[10] Rust, D. M., "Integrated dual imaging detector," (Aug. 1 1995). US Patent 5,438,414.

[11] Mihoubi, S., Lapray, P.-J., and Bigué, L., "Survey of demosaicking methods for polarization filter array images," Sensors 18(11) (2018).

[12] Tyo, J. S., "Optimum linear combination strategy for an n-channel polarization-sensitive imaging or vision system," J. Opt. Soc. Am. A 15(2), 359-366.

[13] LeMaster, D. A. and Hirakawa, K., "Improved microgrid arrangement for integrated imaging polarimeters," Opt. Lett. 39(7), 1811-1814.

[14] Bass, M., Van Stryland, E. W., Williams, D. R., and Wolfe, W. L., [Handbook of optics], vol. 2, McGraw-Hill New York (1995).

[15] Powell, S. B. and Gruev, V., "Calibration methods for division-of-focal-plane polarimeters," Opt. Express 21(18), 21039-21055.

[16] Myhre, G., Hsu, W.-L., Peinado, A., LaCasse, C., Brock, N., Chipman, R. A., and Pau, S., "Liquid crystal polymer full-stokes division of focal plane polarimeter," Opt. Express 20(25), 27393-27409.

[17] Chen, Z., Wang, X., and Liang, R., "Calibration method of microgrid polarimeters with image interpolation," Appl. Opt. 54(5), 995-1001.

[18] Zhang, J., Luo, H., Hui, B., and Chang, Z., "Non-uniformity correction for division of focal plane polarimeters with a calibration method," Appl. Opt. 55(26), 7236-7240.

[19] Han, G., Hu, X., Lian, J., He, X., Zhang, L., Wang, Y., and Dong, F., "Design and calibration of a novel bio-inspired pixelated polarized light compass," Sensors 17(11), 2623 (2017).

[20] Hagen, N., Shibata, S., and Otani, Y., "Calibration and performance assessment of microgrid polarization cameras," Optical Engineering 58(8), 082408 (2019).

[21] Roussel, S., Boffety, M., and Goudail, F., "Polarimetric precision of micropolarizer grid-based camera in the presence of additive and poisson shot noise," Opt. Express 26(23), 29968-29982.

[22] Lapray, P.-J., Thomas, J.-B., and Gouton, P., "High dynamic range spectral imaging pipeline for multispectral filter array cameras," Sensors 17(6) (2017). 